\title{
Heteroassociates of Pinacianol Cation in Aqueous Solutions: Formation and their Interaction with Organic Multiply Charged Anions ${ }^{1}$
}

\author{
Sergey A. Shapovalov ${ }^{a^{*}}$, Yana A. Svishchova ${ }^{b}$ \\ ${ }^{a}$ Research Institute of Chemistry, V N. Karazin Kharkiv National University, \\ Svobody Sq., 4, Kharkiv 61022, Ukraine \\ b Department of General Chemistry, V. V. Dokuchaev Kharkiv National Agrarian University, \\ p/a “Dokuchaevsk - 2”, Kharkiv region 62483, Ukraine \\ serghey.a.shapovalov@,karazin.ua
}

Keywords: heterogeneous associates, dyes, humus acids, pinacyanol chloride, arsenazo II, association constant.

The formation of heterogeneous associates has been studied between the cation of the cyanine dye as pinacyanol chloride (PNC) and multiplies charged anions arsenazo (AR II) or humus acids (HA) in aqueous solutions. The accompanying spectral changes have been analyzed. The equilibrium constants of association $\left(K_{\mathrm{as}}\right)$ were determined, and $\log K_{\text {as }}$ values were equal in the range 1.73-3.09 for associates PNC with HA. The effect of humus acids on $\mathrm{PNC}_{4}^{+} \cdot \mathrm{AR} \mathrm{II}^{4-}$ causes to destruction of the associate and the competition of the anions of HA with AR II is observed.

1 The manuscript is based on the report: Shapovalov S. A., Svishchova Y. A. «Heterogeneous Associates of Organic Dyes: Application to the Analysis of Natural Objects», IX International Conference in Chemistry Kyiv-Toulouse (ICKT-9), 4-9 June 2017, Kyiv, Ukraine. 


\section{Introduction}

The formation of heterogeneous associates of a certain stoichiometric composition was established by a detailed study of the interaction of colored organic counterions in solutions [1]. It was found out that the composition of heterogeneous associates depends not only on the value of charges of counterions, but also on their spatial structure. A heterogeneous association involving cyanine dyes is of particular interest. Such dyes have a well-developed $\pi$-electron system. Many of them have a flattened configuration, which favours association, due to the enhancement of dispersion interactions between molecules. Electronic absorption spectra of dyes change significantly when associates are formed. The additivity of light absorption is disturbed. In some cases, hypsochromic or bathochromic shifts of the bands occur and even new absorption bands can appear. Stability of associates is traditionally estimated using the equilibrium association constant $\left(K_{\mathrm{as}}\right)$ determined by the law of acting masses. For the associates of cyanine dyes, the values of $\log K_{\mathrm{as}}$ were in the range from 2.15 to 13.62 [1].

Supramolecular heteroassociates of dyes are very important to study due to their practical use in chemical analysis and the study of properties of natural objects. In addition, with the development of industry and agriculture it is becoming increasingly important to study the association of dyes with polyelectrolytes (PEL).
The results obtained by this time mainly refer to artificial polyelectrolytes [2]. The study of intermolecular association involving dyes and natural PEL will broaden the understanding of the mechanism of supramolecular interactions. Humus acids (HA) were chosen as PEL, because their structure and composition are an important indicator of humus production processes in soils of different genesis [3, 4]. The aim of the work is to establish the possibility of using a heterogeneous association involving cationic dyes to evaluate the structure of HA. Such studies have not previously been carried out.

\section{Experimental part}

Pinacyanol chloride (PNC) dye and arsenazo II (AR II) (Figure 1) were used as the dyes.

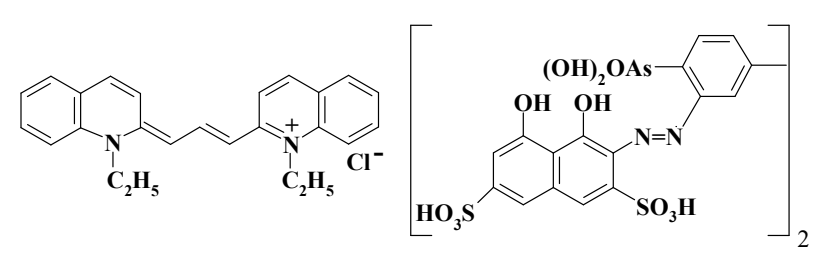

Figure 1. PNC (left) and AR II structures.

The preparation of PNC was of the trade mark "Sigma" and the AR II preparation was of the trade mark "Reanal Labor" with the contents of the main substance are $95 \%$. The initial solutions of AR II with a concentration of $10^{-3} \mathrm{~mol} / \mathrm{l}$ were prepared by dissolving the exact sample. Dilutions of dye solutions with concentrations at $7 \cdot 10^{-7}-5 \cdot 10^{-5} \mathrm{~mol} / 1$ were used for the measurements. The ionic strength of solutions (I) was in the range 0.001-0.008 mol/l. Buffer solutions were used with pH 6.9 and 9.2 
to create the necessary $\mathrm{pH}$. Soil samples of typical black chernozem, deep or middle loam and forest loam with various anthropogenic loads from research areas near V. V. Dokuchaev Kharkiv National Agrarian University (virgin land, arable land, dendropark, greenhouse) and steppe reserve Mikhaylivska virgin land (virgin land, dendropark) have been investigated. From the soil samples, a mixture of HA was isolated by a standard method. Extraction of the preparations was carried out by treating the soil with sodium hydroxide solution at a concentration of $0.1 \mathrm{~mol} / 1$ (the ratio of "soil:solution" is 1:50) after preliminary decalcination with $\mathrm{Na}_{2} \mathrm{SO}_{4}$ solution. Ground suspensions were left a day before filtration. The filtering was carried out after the addition of a saturated solution of $\mathrm{Na}_{2} \mathrm{SO}_{4}$ to the suspension to coagulate the clay particles [5]. Absorption spectra were measured directly after solution preparation against water at room temperature and on a Hitachi-U3210 spectrophotometer in the wavelength range 400$700 \mathrm{~nm}$. Absorption layer length was $1 \mathrm{~cm}$.

Electrical conductivity of water soil extracts measured by coductometrically. Ratio of soil to water was 1:5. Constant of conductometric cell was determined with using of potassium chloride with a concentration of $0.01 \mathrm{~mol} / \mathrm{dm}^{3}$. The change in temperature was taken into account when performing the final calculations.

\section{Results and discussion}

Interactions between PNC and AR II

First of all, let us analyze the possibility of formation of heteroassociates between the PNC cation and multiply AR II anions.

The PNC cation does not change its spectral characteristics in solution at concentrations $7 \cdot 10^{-7}-3 \cdot 10^{-6} \mathrm{~mol} / 1$ and $\mathrm{pH}$ from 3 to 10 , including in the presence of small additions $\left(1 \cdot 10^{-6}-5 \cdot 10^{-5} \mathrm{~mol} / \mathrm{l}\right)$ of inorganic cations or anions. At a $\mathrm{pH}$ greater than 10, the cation is significantly hydrolyzed. As a result, the conjugation chain of polymethine chromophore is disrupted and the intensity of light absorption decreases. At the same time, the solution of PNC partially discolored [6]. Cation PNC due planar structure delocalized charge and is able to form a homogeneous associates (self-associates) at low concentrations. The flat structure of the chromophore system and the delocalization of the positive charge facilitate the interaction of the cations of the PNC with each other. The formation of dimers is noticeable at $7 \cdot 10^{-6} \mathrm{~mol} / 1$ and water-insoluble aggregates at $5 \cdot 10^{-5} \mathrm{~mol} / \mathrm{l}$ or more. The most intense absorption band of the monomeric form has a maximum of $600 \mathrm{~nm}$ ( $\alpha$-band). A band with $550 \mathrm{~nm}$ predominates for the dimer $(\beta-$ band) [6].

Protolytic properties AR II can be estimated by analyzing data on the dissociation constants and the distribution of mole fractions AR I and AR III as a function of $\mathrm{pH}$ [7]. AR II 
has in its composition two hydroxyarsenic groups, four sulfo- groups and four gydroxygroups. Thus, as a result of dissociation, AR II can form quite a lot of anions depending on the $\mathrm{pH}$ of the solution. The dissociation of oxonaphthol fragments occurs in a strongly alkaline medium (at $\mathrm{pH} 10$ or more), and AR II predominantly exists in the form of a multiply charged anion $\mathrm{H}_{4} \mathrm{R}^{8-}$ at $\mathrm{pH} 9.2$ [7] due to dissociated of hydroxyarsenic and sulfo-groups.

The dynamics of spectral changes is most conveniently observed when solutions are mixed with the same concentration of PNC and gradually increasing concentrations of AR II. Changes in light absorption are fixed even with very small additions of AR II when the AR II content at 10 times lower than the PNC. It is noteworthy that the light absorption decreases for both bands of the PNC ( $\alpha$ - and $\beta$-bands), which demonstrates the nonadditivity of light absorption. This fact indicates intense interaction between PNC and AR II (Figure 2).

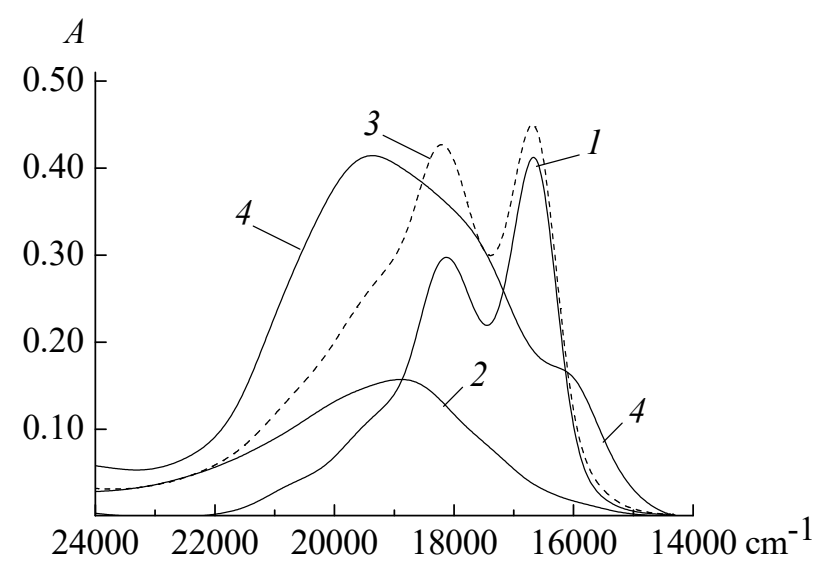

Figure 2. Light absorption in the «PNC+AR II» system. 1 - PNC $\left(8.4 \cdot 10^{-6} \mathrm{~mol} / \mathrm{l}\right) ; 2$ - AR II $\left(1.0 \cdot 10^{-5} \mathrm{~mol} / \mathrm{l}\right) ; 3$ algebraic sum of 1 and 2 spectra; 4 - an experimental spectrum "PNC+AR II" system; pH 9.2.
A feature of the spectral changes is that the «saturation» regions are detected in the light absorption, when the absorption spectrum does not change even with an anion concentration increase. In this case «saturation» occurs when the AR II concentrations are $1.43 \cdot 10^{-6}$ $1.73 \cdot 10^{-6} \mathrm{~mol} / \mathrm{l}$ and the spectrum of the heteroassociate can be directly determined. The band of light absorption of the heteroassociate is hypsochromically shifted relative to the bands of PNC or AR II (Figure 2). The value of $\lambda_{\max }$ is $530 \mathrm{~nm}$ for AR II at $\mathrm{pH}$ 9.2, whereas $\lambda_{\max }$ is $517 \mathrm{~nm}$ for heterogeneous associate of PNC with ARII. The stoichiometric ratio PNC:ARII is determined by analogy with [6]. This ratio is $4: 1$, that is, one eight-charged anion AR II coordinates four cations of the cyanine dye. Thus, an associate $\left(\mathrm{PNC}^{+}\right)_{4} \cdot \mathrm{AR} \mathrm{II}{ }^{8-}$ is formed in the simplest case with an uncompensated negative charge. Apparently, combining more cations of PNC near one anion is difficult due to steric hindrances.

The Adamovich method was used to evaluate the stability of associates with respect to the study of equilibrium $\left\langle\mathrm{PNC}^{+}+\mathrm{AR} \mathrm{II}^{8-}\right\rangle$ [8]. As is known, the formation of associates between organic ions has a step-like character. The presence of one isosbestic point in the spectral series and one «saturation» region indicates the formation of associates of a certain stoichiometry. In this case it is an associate $\left(\mathrm{PNC}^{+}\right)_{4} \cdot \mathrm{AR} \mathrm{II}^{8-}$. The value of the concentration 
constant $\log K_{\text {as }}=2.15 \pm 0.01$ was obtained for equilibrium:

$$
4 \mathrm{PNC}^{+}+\mathrm{AR} \mathrm{II}^{8-} \rightleftarrows\left(\mathrm{PNC}^{+}\right)_{4} \cdot \mathrm{AR} \mathrm{II}^{8-} .
$$

The ionic strength of the solutions is small because of the concentrations of the interacting particles are low. Therefore, the equilibrium concentrations of dyes practically coincide with the activities, and the concentration constant $K_{\text {as }}$ coincides numerically with the thermodynamic one. A comparatively small value of the association constant can be explained by the fact that the anion is not a planar structure. This prevents the close location of the counterions and weakens the dispersion interactions between the chromophores, which are significant in the interactions of polyatomic supramolecular structures.

Interactions between PNC and PEL as also between PNC·AR II and PEL

The interaction of polyatomic anions with cationic dyes can lead not only to the formation of heteroassociates. For example, in the presence of a multiply charged anion, PNC cations are able to form self-associates and even H-type aggregates. The effect of synthetic PEL on the formation of self-associates was studied earlier. It was found that the intensity of induction of associates depends on the ratio of the amounts of the dye and PEL [1]. Natural PEL are humus acids (HA). They are a mixture of nitrogen-containing macromolecular compounds. They contain in their composition hydrolized and non-hydrolyzed fragments. Hydrolized fragments are branched and mainly have carbonyl, carboxyl, hydroxyl and phenolic functional groups. Non-hydrolyzed fragments contain aliphatic and aromatic nuclei. The degree of recovery and composition of HA depend on many factors, such as extraction conditions, reagent type, solution concentration, contact time with solution, amount of extrusion, temperature, soil volume and solution ratio [9]. We did not separate humus acids HA into separate fractions (humic acids and fulvo acids), since it is multi-stage and often accompanied by irreversible changes in the initial chemical composition. For these reasons, the changes in spectral characteristics under discussion should be attributed to the influence of all fractions of HA.

The absorption spectra of humus acids have the form of shallow curves with a maximum absorption band in the short-wave part of the UV spectrum. Interpretation of the spectra is reduced mainly to the calculation of the extinction coefficient $\left(\mathrm{E}_{465 / 650}\right)$. This value makes it possible to characterize the structural features of humus acids, such as the type of soil, the method of soil cultivation. The method of investigation involves bringing the optical density of solutions of various soil humbs substances to the same carbon concentration. However, the informativity of such spectrum may decrease due to the dilution effect [10]. 
The character of the absorption spectra of studied HA in the visible region is of the same type. Spectra show a monotonic increase in absorption in the region of short wavelengths and do not have characteristic peaks. Despite of the spectrophotometric method limitations the interpretation of the obtained spectra indicates different values of the extinction coefficients. We have analyzed the values of the extinction coefficients for $\lambda=465 \mathrm{~nm}$ and $\lambda=650 \mathrm{~nm}\left(\varepsilon_{4}\right.$ and $\varepsilon_{6}$, respectively). The $\varepsilon$ values were used to calculate the HA concentrations (Table 1). From these data it follows that the higher the values of the extinction coefficients, the greater the proportion of unhydrolyzed fragments of the aromatic system in comparison with the functional groups of peripheral chains in HA.

Table 1. The humus content in the soil and extinction coefficients of soil extracts

\begin{tabular}{|l|c|c|c|}
\hline $\begin{array}{c}\text { Soil extracts } \\
\text { from }\end{array}$ & $\begin{array}{c}\text { The humus } \\
\text { content, } \%\end{array}$ & $\varepsilon_{4}$ & $\varepsilon_{6}$ \\
\hline greenhouse $^{1}$ & 4.20 & 0.064 & 0.007 \\
\hline arable land $^{1}$ & 4.60 & 0.183 & 0.021 \\
\hline dendropark $^{1}$ & 5.12 & 0.234 & 0.031 \\
\hline virgin land $^{1}$ & 5.02 & 0.214 & 0.025 \\
\hline dendropark $^{2}$ & 7.67 & 0.275 & 0.037 \\
\hline virgin land $^{2}$ & 7.19 & 0.409 & 0.051 \\
\hline
\end{tabular}

${ }^{1}$ Soil samples are taken from research areas near V. V. Dokuchaev Kharkiv National Agrarian University; ${ }^{2}$ soil samples are taken from Mikhaylivska virgin land.

Spectral changes become more significant with an increase in the volumes of the soil extract of Mikhaylivska virgin land, which are added to a fixed amount of PNC (Figure 3).

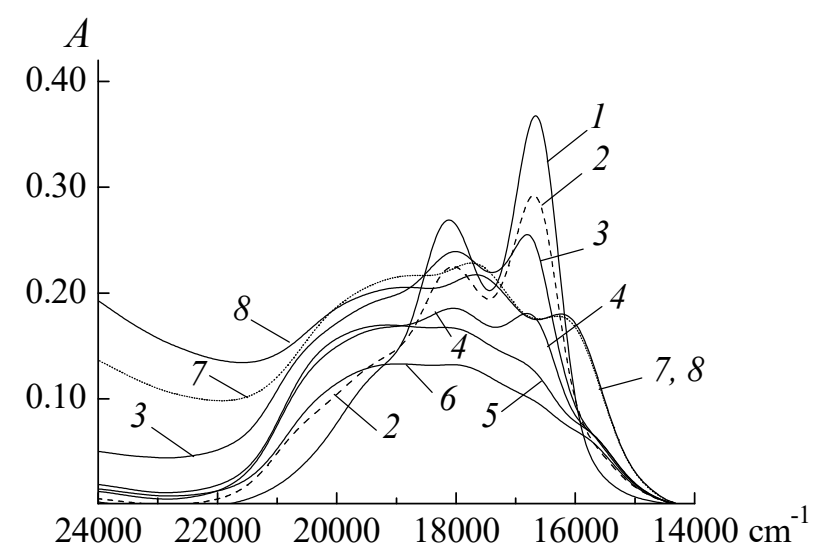

Figure 3. Change in light absorption of PNC under adding of the soil extract of Mikhaylivska virgin land. Concentration, mol/1, of PNC: $3.8 \cdot 10^{-6}$; of the soil extract: $1-0,2-3.1 \cdot 10^{-4} ; 3-6.2 \cdot 10^{-4} ; 4-9.3 \cdot 10^{-4} ; 5-2.1 \cdot 10^{-3}$; $6-3.1 \cdot 10^{-3} ; 7-2.1 \cdot 10^{-2} ; 8-4.2 \cdot 10^{-2} \cdot \mathrm{pH} 6.8$.

The intensity of absorption decreases by $23 \%$ when the concentration of $\mathrm{HA}$ is $3.1 \cdot 10^{-4} \mathrm{~mol} / \mathrm{l}$. A further decrease in the optical density and a change in the shape of the absorption band occur with an increase of the HA concentration up to $2.1 \cdot 10^{-3} \mathrm{~mol} / \mathrm{l}$. The saturation of $A$ is reached at a concentration of $3.1 \cdot 10^{-3} \mathrm{~mol} / \mathrm{l}$, and a maximum of the absorption band $565 \mathrm{~nm}$ appears at even higher concentrations $4.2 \cdot 10^{-2} \mathrm{~mol} / 1$. Similar changes were also observed when other soil extracts were added to the PNC solution. New absorption maximum 563-565 nm appears at HA concentrations of $7.6 \cdot 10^{-3}-6.1 \cdot 10^{-2} \mathrm{~mol} / 1$. Analysis of spectral changes similar to Figure 3 indicates intensive interaction of the cyanine dye with HA. Reduction of light absorption of $\alpha$ - and $\beta$-bands of PNC was observed in all cases. But the formation of dimers and more complex aggregates of cyanine dye does not 
occur. The absence of a self-association can be explained by the fact that the PNC cations on the HA surface are located at rather large distances from one another, and cation-cation type interaction does not occur. However, despite the similarity of the spectral changes for all soils, there are differences in the influence of HA on the absorption of the PNC cation.

As can be seen from the data in the Table 2, spectral changes are observed in extracts for soils with a high content of humus. The magnitude of changes in the optical density depend on the degree of benzoic acidity of the HA. For soil extracts of greenhouse which have the least value of extinction coefficients (Table 1), the saturation region is observed at concentrations of HA $3.12 \cdot 10^{-2} \mathrm{~mol} / \mathrm{l}$.

Table 2. The change in the optical density of PNC with the addition of HA and the electrical conductivity, $\chi$, of water extracts of the soil

\begin{tabular}{|l|c|c|c|}
\hline \multicolumn{1}{|c|}{ Soil extracts from } & $\Delta A$ & $\mathrm{C}(\mathrm{HA}), \mathrm{mol} / \mathrm{l}$ & $\begin{array}{c}\chi, \\
\mu \mathrm{S} / \mathrm{cm}\end{array}$ \\
\hline greenhouse $^{1}$ & 0.16 & $4.7 \cdot 10^{-3}$ & 676 \\
\hline arable land $^{1}$ & 0.16 & $3.4 \cdot 10^{-3}$ & 86.9 \\
\hline dendropark $^{1}$ & 0.17 & $2.5 \cdot 10^{-3}$ & 95.0 \\
\hline virgin land $^{1}$ & 0.15 & $2.3 \cdot 10^{-3}$ & 33.0 \\
\hline dendropark $^{2}$ & 0.16 & $6.5 \cdot 10^{-3}$ & 109 \\
\hline virgin land $^{2}$ & 0.15 & $6.2 \cdot 10^{-4}$ & 68 \\
\hline
\end{tabular}

${ }^{1}$ Soil samples are taken from research areas near V. V. Dokuchaev Kharkiv National Agrarian University; ${ }^{2}$ soil samples are taken from Mikhaylivska virgin land.

Thus, the stabilization of associates occurs due to the electrostatic and dispersion interaction between $\pi$-electronic of PNC-HA systems.

Table 2 shows the electrical conductivity of water extracts of the soil. As is known [11], the value of electrical conductivity is an indicator of the relative content of inorganic ions in the soil. For water extracts of areas near the agrarian university the soil of virgin land is less saline than the soil of the dendropark. Soils of Mikhaylivska virgin land have similar patterns in salinity. As can be seen from the Table 2 data, the same spectral changes are observed at different levels of salinity.

The interaction between PNC and HA can be represented in the form:

$$
n P N C^{+}+H A^{m-} \rightleftarrows\left(P N C^{+}\right)_{n} H A^{m-}
$$

then $K_{\text {as }}$ is in the form:

$$
K_{\text {as }}=\frac{\left[\left(P N C^{+}\right)_{n} H A^{m-}\right]}{\left[P N C^{+}\right]^{n} \cdot\left[H A^{m-}\right]}
$$

$\left[\left(P N C^{+}\right)_{n} H A^{m-}\right],\left[P N C^{+}\right],\left[H A^{m-}\right]$ are equilibrium molar concentrations of associate, of PNC, and of HA, respectively.

Equations of material balance are:

$$
\begin{gathered}
{\left[P N C^{+}\right]_{\mathrm{o}}=\left[P N C^{+}\right]+n \cdot\left[\left(P N C^{+}\right)_{n} H A^{m-}\right],} \\
{\left[H A^{m-}\right]_{\mathrm{o}}=\left[H A^{m-}\right]+\left[\left(P N C^{+}\right)_{n} H A^{m-}\right],} \\
\text { Imagine the optical density as: }
\end{gathered}
$$

$$
A_{600}=\left(\varepsilon_{1} \cdot\left[P N C^{+}\right]+\varepsilon_{2} \cdot\left[\left(P N C^{+}\right)_{n} H A^{m-}\right]\right) \cdot l
$$

$\varepsilon_{1}$ and $\varepsilon_{2}$ are extinction coefficients of $\left[P N C^{+}\right]$ and $\left[\left(P N C^{+}\right)_{n} H A^{m-}\right], l$ is length of optical path, []$_{\mathrm{o}}$ are initial molar concentrations.

Considering all the above: 
$\frac{l}{\varepsilon_{1} \cdot\left[P N C^{+}\right]_{0}-A}=\frac{1}{K_{a s} \cdot\left[P N C^{+}\right]_{0} \cdot\left(\varepsilon_{1}-\varepsilon_{2}\right)} \cdot \frac{1}{\left[H A^{m-}\right]_{0}}+$ $+\frac{1}{\left[P N C^{+}\right]_{0} \cdot\left(\varepsilon_{1}-\varepsilon_{2}\right)}$.

The coefficient of extinction of $\left(P N C^{+}\right)_{n} H A^{m-}$ was calculated on the basis of linearization of the experimental data in coordinates $\frac{1}{\varepsilon_{1} \cdot\left[P N C^{+}\right]_{0}-A} \div \frac{1}{\left[H A^{m-}\right]_{0}}$ it's a given that $l=$ $1 \mathrm{~cm}$. The $K_{\text {as }}$ values were calculated on the basis of founded extinction coefficients.

The obtained values of $\varepsilon$ and $\log K_{\text {as }}$ are presented in Table 3.

Table 3. The values of the extinction coefficients of the associates and the values of the constants of association

\begin{tabular}{|l|c|c|}
\hline \multicolumn{1}{|c|}{$\begin{array}{c}\text { Soil extracts } \\
\text { from }\end{array}$} & $\varepsilon$ & $\log K_{\mathrm{as}}$ \\
\hline greenhouse $^{1}$ & 29263 & $1.73 \pm 0.12$ \\
\hline arable land $^{1}$ & 25210 & $2.12 \pm 0.08$ \\
\hline dendropark $^{1}$ & 20787 & $2.45 \pm 0.05$ \\
\hline virgin land $^{1}$ & 20210 & $2.49 \pm 0.03$ \\
\hline dendropark $^{2}$ & 15981 & $2.63 \pm 0.12$ \\
\hline virgin land $^{2}$ & 10739 & $3.09 \pm 0.03$ \\
\hline
\end{tabular}

${ }^{1}$ Soil samples are taken from research areas near V. V. Dokuchaev Kharkiv National Agrarian University; ${ }^{2}$ soil samples are taken from Mikhaylivska virgin land.

The $\log K_{\text {as }}$ values are 2-4 times and 1.5 times less than for the associates, respectively, of doubly charged and singly charged anions of sulfonephthaleins and oxyxanthenes with PNC [12].

Associate with greenhouse has the lowest $\log K_{\text {as }}$ value (1.73 \pm 0.12$)$ (so, in the composition of humus acids there are functional groups of peripheral chains). On the contrary, the largest of $\log K_{\text {as }}$ value $(3.09 \pm 0.03)$ has an associate with HA of the Mikhailovsky virgin land (humus acids contain fragments of nonhydrolyzed aromatic systems).

We have studied the effect of HA on the associate $\left(\mathrm{PNC}^{+}\right)_{4} \cdot \mathrm{AR} \mathrm{II}^{8-}$. For this, $\mathrm{PNC}$ and AR II were used at concentrations $8.4 \cdot 10^{-6}$ and $1.0 \cdot 10^{-5} \mathrm{~mol} / 1$, respectively. The association is most noticeably manifested with these concentrations. A bathochromic shift of maximum of absorption band is observed with 517 to $565 \mathrm{~nm}$ when $\mathrm{HA}$ is added under concentrations of $0.023-0.055 \mathrm{~mol} / \mathrm{l}$. Also, a decrease in the intensity of light absorption is observed if additives of HA with $\varepsilon_{4}=0.214$ 0.409 are added to the mixture of PNC and AR II. On the contrary, an increase in the intensity of light absorption occurs if the HA additives have $\varepsilon_{4}=0.064-0.183$. The observed spectral changes can be explained by the destruction of the associate $\mathrm{PNC}_{4} \cdot \mathrm{AR} \mathrm{II}^{4-}$ and the formation of PNC-HA associate with $\lambda_{\max }=$ 563-565 nm of light absorbance band. Figure 4 shows the spectral changes when soil extract of dendropark in the Mikhaylivska virgin land (HA) is added to the «PNC+AR II» system. 


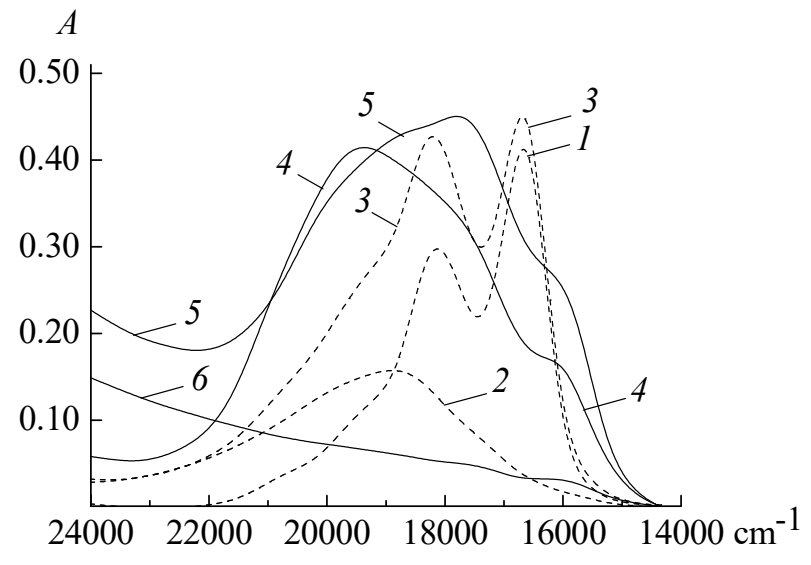

Figure 4. Light absorption in the «PNC+AR II+HA» system. $1-\quad \mathrm{PNC}\left(8.4 \cdot 10^{-6} \mathrm{~mol} / \mathrm{l}\right) ; 2-$ AR II $\left(1.0 \cdot 10^{-5} \mathrm{~mol} / \mathrm{l}\right) ; 3$ - algebraic sum of 1 and 2 spectra; $4-$ an experimental spectrum «PNC+AR II» system; 5 adding $0.023 \mathrm{~mol} / \mathrm{l}$ of HA to 4 ; 6 - HA ( $0.023 \mathrm{~mol} / \mathrm{l}) . \mathrm{pH}$ 9.2 .

The destruction of $\mathrm{PNC}_{4} \cdot \mathrm{AR} \mathrm{II}^{4-}$ associate occurs under sufficiently high concentrations of HA (spectrum 5). The obtained results make it possible to assume that the multiply charged PEL anions «compete» with the AR II anions according to the scheme: $\left(\mathrm{PNC}^{+}\right)_{4} \cdot \mathrm{AR} \mathrm{II}{ }^{8-}+\mathrm{HA}^{\mathrm{n}-} \rightarrow \mathrm{PNC}^{+} \cdot \mathrm{HA}^{\mathrm{n}-}+\mathrm{AR} \mathrm{II}^{8-}$

\section{Conclusions}

Two directions of the formation and use of heteroassociates have been discussed. The first direction is based on recording changes in the absorption spectra of protolytic forms of charged cyanines when they interact with organic counterions in solution. The second direction is based on spectral changes, when the voluminous ion replaces one of the counterions in the heteroassociate. Thus, there is competition between ions to form the most stable associated substance. Such types of interactions may be useful for developing methods for quantifying the concentration of PEL of natural origin, such as humus acids. The stoichiometry of heteroassociates with the participation of multiply charged organic ions depends on the structure of counterions. Addition of high concentrations of HA causes destruction of the heteroassociate $\left(\mathrm{PNC}^{+}\right)_{4} \cdot \mathrm{AR} \mathrm{II}{ }^{8-}$ due to competition between PEL and anions of AR II.

\section{References}

[1] Shapovalov SA. The association processes of protolytic forms of dyes in solutions. Dissimilar association. Kharkiv: Brovin Publishing house; 2014, 250 P.

[2] Mamleeva NA, Pisareva TP. Association of lignin with methylene blue in aqueous solutions. J. Phys. Chem. 1994; 69(3): 504-509.

[3] Karabaev SO, Lokshina IP. Isolation and analysis of humic acid from coal Kara-keche. Bulletin of the KRSU. 2010; 10(10): 161-164.

[4] Nesterova OV, Semal VA, Characterization of humic acids of the Southern brown soils Sikhote-Alin by the data of element analysis and IR spectrometry (on the example of the Ussuriisk reserve). Bulletin of the KRSU, Series Soil science. 2009; 10: 29-35.

[5] Orlov D. Workshop on the chemistry of humus. Moscow: Moscow State University; 1981, 272 P.

[6] Svishchova YA, Shapovalov SA. Interactions between xylenol orange and pinacyanol in aqueous solutions. Kharkov University Bulletin. Series Chemistry. 2012; 1026(21): 9-11.

[7] Ivanov VM, Ermakova NV. Optical and colorimetric characteristics of erbium complexes with 
arsenazo I, arsenazo III and hlorfosfonazo III. Bulletin of Moscow State University. Series Chemistry. 2000; 41(3): 174-177.

[8] Bulatov M.I., Kalinkin I.P. A practical guide to photometric methods of analysis. Leningrad: Chemistry; 1986, 432 P.

[9] Orlov D. Soil chemistry. Moscow: Moscow State University; 1992, 400 P.

[10] Kudeyarova AYu. The use of electronic spectroscopy to detect structural differences between humic acids of virgin and arable gray forest soil. Pochvovedenie. 2008; 9: 1079-1091.

[11] Kalvoda R. Electrochemical methods in environmental control. Moscow: Chemistry; 1990, 238 P.

[12] Shapovalov S., Svishchova Y. Association of pinacianol with anions of sulfonephthalein and oxyxanthene dyes in aqueous solutions. Ukr. Chem. J. 2002; 68(4): 107-110. 